\title{
Use of software analytics of brain MRI (with \& without contrast) as objective metric in neurological disorders and degenerative diseases
}

\begin{abstract}
Complex degenerative and inflammatory processes of the central nervous system (CNS) often feature certain lymphocytic infiltrates and changes, which are felt to be involved in visual image alterations correlating with damage of myelin and nerve tissues per se. Changes are thought to be associated with autoimmune and degenerative disorders within the CNS often are demonstrated by changes on magnetic resource imaging (with and without contrast). These imaging changes have proven to demonstrate specific and progressive changes which may be correlated with failure of myelination resulting in a wide variety of non-functional clinical states and symptoms.

Such entities, including stroke, traumatic brain injuries (TBI), complex regional pain syndromes (CRPS), and tumors often present chronic and persistent disabilities. Use of quality imaging and interpretation is considered of great value to patient management. Progression and severity can now be accurately tracked utilizing the novel diagnostic software where detailed locations, volumetric analytics, lesion regression-progression monitoring etc. are available. Traditional interpretations are often tedious, and result in highly variable, and may provide incomplete comparative reporting. Inconsistent findings can result in limited or only moderately effective diagnostics using imaging interpretation by highly trained imaging specialists. The issue of standardization, depth, quality consistent interpretation and provision of such detail is an expensive and elusive challenge, making comparatives and interpretation somewhat limited.

Time consumption, limited accuracy, and costs of serial MRI segmented/subsegmented analytics make very detailed, accurate neuroradiological interpretations almost impossible to standardize. Frequently different imaging specialist are often used for examining serial studies, thereby potentially magnifying potential variables. Use of a new, highly sophisticated, software helps resolve many issues, including taking manual (human) interpretation out of potential bias or different interpreters, while providing serial ability to track lesion and volumetric analysis in evaluation and management of these progressive and often debilitating circumstances. This paper intends to introduce this European technological advance to the North American and world marketplace, where few have seen the opportunity to take advantage of this software technology, and gain the opportunity to standard interpretations from a diagnostic and ongoing management standpoint.
\end{abstract}

Keywords: brain MRI, neurological disorders, neurological degeneration, MS, TBI, Stroke, parkinson's, dementia, alzheimer's, CRPR
Volume 2 Issue 2 - 2017

Robert W Alexander

Department of Surgery, University of Washington, USA

Correspondence: Robert W Alexander, Department of Surgery, University of Washington, School of Medicine and Dentistry, USA, Tel + | 4067774477, Fax + |8667665458, Email rwamd@cybernetl.com

Received: September 27, 2017 | Published: November 17, 2017

\section{Overview of neurodegenerative applications}

As example, we present use of the PIXYL software in a case of Multiple Sclerosis (MS), a frequently occurring inflammatory, demyelinating, and autoimmune disorder of the CNS. ${ }^{1-3}$ Specifically, white matter tracts are involved, including cerebral, infra-tentorial, and spinal cord area. Lesions, referred to as "plaques" are often visible on MRI (with and without contrast), and pose a challenge to diagnosis, interpret, or track effects of medications or cellular-based therapies $^{4,5}$ (Figure 1) (Figure 2).

MRI has revolutionized the diagnosis and monitoring of such patients. Its sensitivity and interpretations are enhanced with use of contrast media, making early evaluations in patient's even with a single episode of neurologic impairment who do not meet the clinical criteria for diagnosis. ${ }^{6}$ Use of 3-dimensional (3-D), T2* weighted, gradientecho $((\mathrm{T} 2 * \mathrm{GRE})$ and white matter-attenuated, turbo-field-echo (TFE) sequences at $7 \mathrm{~T}$ field strength is very effective at recording most cortical lesions. ${ }^{7}$ MRI contrast use helps identify variations reflecting alterations in brain iron and myelin., ${ }^{8,9}$

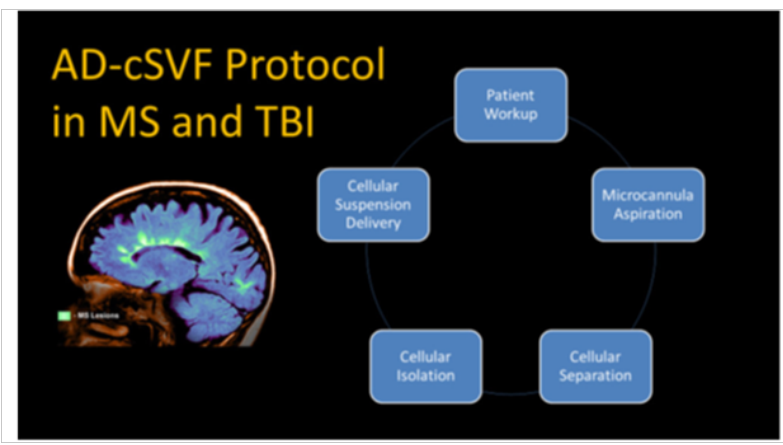

Figure I Clinical Trial Outline of Treatment. 


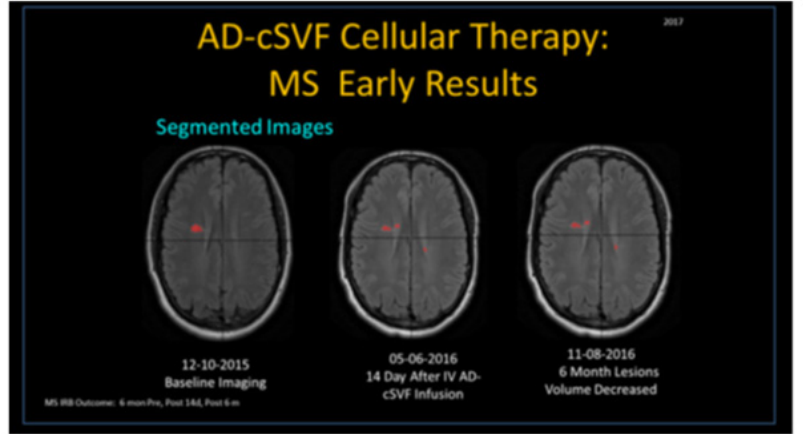

Figure 2 multiple sclerosis (MS) trail patient. left - pre-cellular therapy; middle - two weeks post-cellular infusion; right - six month post-cellular therapy.

Inflammation and breakdown of blood-brain barrier occurs in MS lesions, leading to changes in extravascular fluid produces hyperintensity of the T2-weighted images, reported as hyper-intense white matter lesions. T1-weighted images are seen as dark (hypo-intense) images caused by tissue with more water, while high fat content (white matter) and appear bright. Newer MRI pulse sequences and techniques, such as fluid-attenuated inversion recovery (FLAIR), uses a heavily T2-weighted technique which dampens the ventricular cerebrospinal fluid (CSF) (i.e., free-water). This leaves brain parenchymal lesions (e.g., plaques of MS), leaving the CSF appearance as black. The PIXYL software algorithms are capable of interpretations using these modalities, with accuracy that rivals, or may exceed, manual readings. When comparing a series of MRI in the same patient, this is a huge time-saver and cost effective means of tracking. For those clinical trial investigators who are neither expert in reading, nor radiologically back grounded, this is considered a major contribution.

We present the process as a preliminary example of our clinical trial (NCT \#02939859) wherein we have been tracking the safety and efficacy of use on autologous stem/stromal cell therapy derived by processing adipose tissue complex to isolate and concentrate its cellular stromal vascular fraction (CSVF). After concentration, these cells are suspended in sterile Normal Saline and delivered via intravascular route. With recognition of certain capabilities of the adipose-derived CSVF, including potentially important immune modulation and inflammatory modulation properties, the trial was established to explore potential advantages of uses of autologous, CSVF in neurodegenerative-related clinical problems. In the face of the disorders not being effectively dealt with using traditional pharmacological products, very expensive medications, and a variety of unacceptable side effects ${ }^{10,11}$ (Figure 3 ).

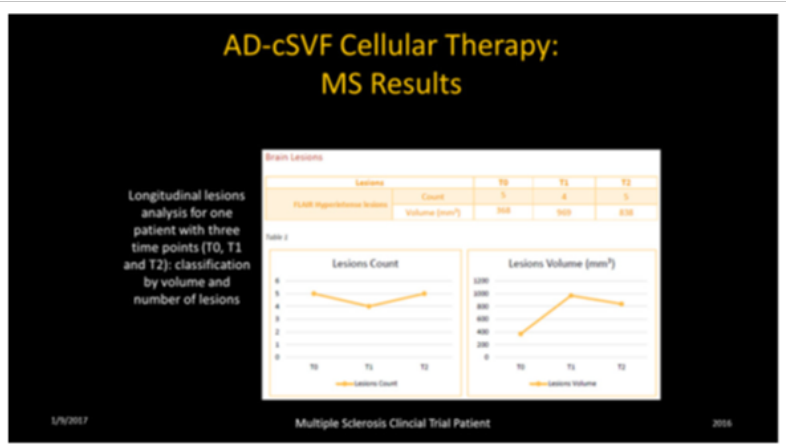

Figure 3 Lesion counts: NOTE: counts initially decline, then return to baseline while the measured volumes were dramatically reduced at six months. Patient clinical progress markedly improved in all categories.
The design of the protocol, includes objective metrics for evaluation of relative points and determined to be tracked via magnetic resonance imaging (MRI with/without contrast). This is an accepted metric in common use amongst neuro-radiologists and neurologists in diagnostic and monitoring applications in management of progressive MS patients. ${ }^{12}$ There is a general reluctance for some neurological specialists to order repetitive MRI due to the limitation of therapeutic alternatives other than diet, physical therapy, and medications. Unfortunately, many of the available medications have very undesirable side effects, and often prove of limited efficacy. When dealing with patient follow up examinations, several specialist that were contacted said the studies were expensive and there was nothing particular they could clinically offer the patients based on the MRI changes even if progression of lesions were noted.

In order to evaluate the potential changes that could be attained with cellular therapy, we needed a consistent method of objectively documenting changes in sizes, locations, or volumes of the lesion (plaques) found. That led to discussions with a European company (PIXYL) who developed by very sophisticated software program specifically capable of repetitive analysis (baseline comparative to 6 month interval sampling), while providing study a high quality interval correlative interpretations. This permitted a consistent ability to accurately "track" lesions, without having to have multiple interval segmenting demands by radiologists in each case. Manual examinations are very time consuming and subject to human error, and it is prohibitively expensive to be practical. The software and its complex mathematical iterations, prove to be consistent, accurate, and considerably less difficult to acquire analyses (Figure 4) (Figure 5).

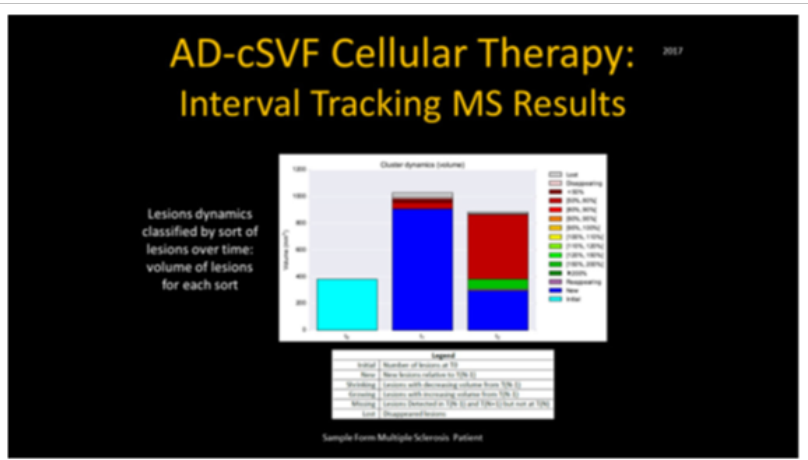

Figure 4 Lesion dynamic at intervals tested (See next Figure image cluster dynamic of changes in lesions).

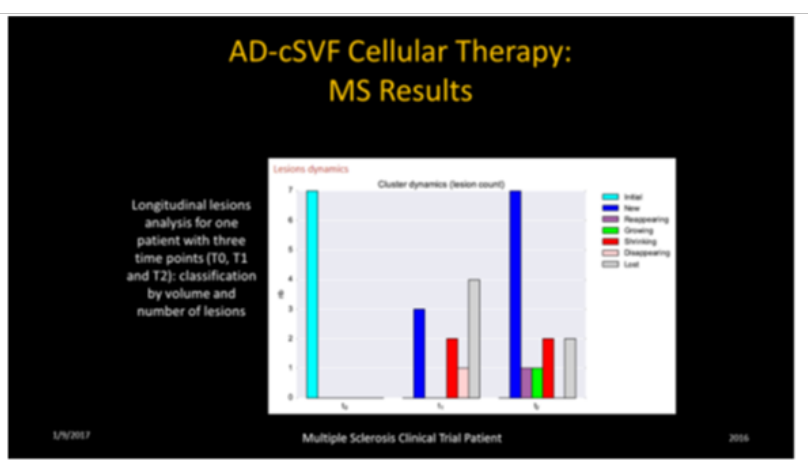

Figure 5 Cluster dynamics (counts and activity) initial, new, reappearing, growing, shrinking, disappearing and lost categories.

These analyses permit location, number, sizing, status of lesions (new, existing, growing, declining, fragmenting (lower volume 
over previous exam), and lost lesions in comparative fashion. It is considered imperative to distinguish a variety of lesions (size, location, texture of each). This is not a simple interpretation due to the variety of aging, vascular changes, locations, and disease process, etc. In development of the software protocols, the assumption was that manual segmentation was the gold standard, and the goal of the PIXYL analysis was to compare to the "ground truth" (true abnormal voxels) to the similarity index and evaluating the ROC curves. The differences in interpretation could be minimized, and the outcome analytics showed an excellent correlation.

The comparison between brain-damaged versus healthy subjects was determined that differences only related to presence or absence of abnormal tissue. These imaging studies can then be used to attempt to cancel or minimize sources of differences by demographic variables (age, gender, etc.) and to insure an accurate segmentation or normalization protocols. ${ }^{13-15}$ Since most clinical trials exploring these subjects are not practical due to logistics, having expert radiologists on staff, or may not be able to reasonably afford repetitive analytics on interval images. Regardless of patient location or clinical availability of expert interpreters, use of this software could provide a reasonable and effective alternative and standardization of comparative exams such as could be offered by PIXYL utilization.

Clinical Trial design and protocols include evaluation of relative points of positive findings on magnetic resonance imaging, and help identifying changes in the neurodegenerative patients. Coupled with standard medical testing, such changes are a means of tracking clinical progress with objective findings of value. As a simplification of our trial, the hypothesis of utilization of CSVF was tested with serial documentation and tracking of changes using the software analysis at 6 month intervals. Interval neurological examination by the patient's primary specialists, observational diary reporting is coupled with interval subjective Quality of Life (QOL) questionnaires.

Simple closed syringe microcannula aspiration from optimal sub dermal deposits of abdomen and flanks (male and female patients) or inner-outer thigh area (females) are gently extracted in volumes of approximately $50 \mathrm{cc}$ of tissue stromal vascular fraction. After sterile technique for acquisition, the tissues are centrifuged to remove extraneous fluids and placed in a Healeon Medical Centricyte 1000(Newport Beach, CA, USA) for enzymatic digestion and cellular separation/concentration devoid of its native matrix. The isolated cells were next rinsed and neutralized using a proprietary process to remove unwanted enzymes. Following final centrifugation, the cellular "pellet" is re-suspended in sterile normal saline and deployed slowly in an intravenous infusion.

The logic of this standardized protocol is to provide evidence of safety and efficacy of such cellular infusions as an alternative to traditional medication limitations, and sequentially evaluate the patient changes in an objective fashion along with the standard neurologist examination of patients at 6 month interval over a five year period. Objective metrics are often difficult to measure, and subjective comments highly variable. Use of the PIXYL software has proven to be a cost effective, reliable, and consistent objective measure in correlation with clinical progress in patients.

\section{Sample utilization in ms patient}

Example of 32 year old progressive MS patient with severe intolerance to all tried medications (severe adverse events requiring hospitalization) was entered into the clinical trial. Treatment included diet, physical therapy, and neurologic tracking. Clinical response was considered remarkable for improvement of existing clinical symptoms, with return of functional deficits clinically, correlated with significant reduction of volumes of plaque lesions and fragmentation of same. Serial MRIs continue to demonstrate objective changes, while clinical return to functions persist at more than one and one-half years.

PIXYL software designed for medical research and practice is designed to consistently extract the maximum information contained in MRI brain scans. Its complex solution specifically is able to locate, identify, and quantify a wide range of lesion changes related to pathology (MS, stroke, variety of degenerative disorders and traumatic brain injuries (TBI) ${ }^{16}$ The tools developed are able to automatically identify and measure healthy brain tissue and lesions, based on densities, without the variability introduced by manual segmentation analysis. Such manual delineations are difficult to re produce, time consuming and very costly needs when using standard interpretations. The software is unique, with its abilities to located region of the lesions, then classify into "sub-regions" to be able to calculate volumes, using statistical modeling algorithms based on advances in neuro imaging, classification and distributive computer. ${ }^{17}$

With proven abilities to accurately track and measure lesion volumetrics in the brain, without the common interpretation variances, the attraction to apply this technology to tracking in clinical trials and practice is obvious. Consistency is very important in these interpretations, making the proven software modeling in a patient advances.

\section{Discussion}

Due the frequent diversity of manifestations associated with MS, and its variants, any improvements of the tracking progress and therapeutic options in these clinical patients is considered of great value. Since many providers are often involved in patient management as a team, the ability to document changes may help in the decision process for optimal patient support and care.

Unfortunately, there is not a single test or examination that is fully diagnostic or effective at stopping, slowing, or improving the neurological conditions from most TBI to degenerative conditions. The traditional options typically are based on revealing asymptomatic dissemination of what matter lesions in space and over time.

To date, the MRI is generally considered the most sensitive method of revealing the pattern and extent of dissemination in space (DIS) and time in space (DIT) has helped with earlier diagnostics, but not how to manage or change the processes. To that end, use of cell-based therapies are offering some encouraging and interesting options in a wide group of neurological patients. Uses of this software will likely play a very important role in diagnostics for the disease as a group, and include alternative issues found in spinal stenosis, stroke, genetic disorders, TBI, recurring concussion changes, trauma, and tumors.

In this example, the characteristic lesions are Dawson Fingers, ovoid lesions (plaques), corpus callosum lesions but often allow evaluation of many cerebellar and upper spinal cord lesions. For many years, rehabilitation and neurological providers have elected to rarely retake MRIs, primarily due to the lack of traditional-type medications that have shown remarkable success at symptom improvement and only some with slowing or helping reduce recurrences. Due to the nature of some of these disorders, long-term tracking is essential, 
and PIXYL software now provides a consistent and reliable analysis. From these standardizations, more accurate analyses of therapeutic modalities can be attained and tracked. ${ }^{18}$

\section{Conclusion}

Use of software analytics may prove of great value, both regards the patients progression, rehabilitation capabilities and options, and for applied clinical research. Neurologists and PM\&R specialists often need to have options for evaluation of effectiveness of physical rehabilitation, diet, exercise progressions (both physical and mental) efforts. The variabilities of findings in manual segmentation/subsegmentation via a single interpreter is significant. The ability to provide serial analytics makes the confidence factor and accuracy grow, may revolutionize our management options.

Doing interval testing and tracking is an essential to determine the efficacy, and eventually optimize the modalities to work together in management of a large variety of patients. In the clinical trial setting, the efficacy of medications and/or cellular therapy combinations can be optimized and tracked. Early observations and clinical correlations have supported a very good safety profile in all patients, with clinically positive changes for registered patient population within the trial. Much is needed in reporting on long-term tracking at this point in time. Outcomes of early patient groups receiving cell-based protocols will most definitely benefit from an objective analysis. Like the example shown briefly, the early changes are being seen in this population where traditional options are so limited.

\section{Acknowledgements}

Acknowledge the essential supportive clinical and laboratory staff directors for their many contributions, without whom the patient management and clinical trial tracking would not be possible. Nancy Smith, KaSandra Towner and Susan Riley are hereby recognized.

\section{Conflict of interest}

The author declares no conflicts of interest.

\section{References}

1. Lukes SA, Crooks LE, Aminoff MJ, et al. Nuclear magnetic resonance imaging in multiple sclerosis. Ann Neurol. 193;13(6):592-601.

2. Hashemi RH, Bradley WG, Chen DY, et al. Suspected multiple sclerosis: MR imaging with a thin-section fast FLAIR pulse sequence. Radiology. 1995;196(2):505-510.

3. Koch MW, Metz LM, Agrawal SM, et al. Environmental factors and their regulation of immunity in multiple sclerosis. J Neurol Sci. 2013;324(12):10-16.
4. Hassan SG, Douglas MR. Management and prognosis of multiple sclerosis. Br J Hosp Med (Lond). 2011;72(11):M174-176.

5. Wattjes MP, Steenwijk MD, et al. MRI in the diagnosis and monitoring of multiple sclerosis: an update. Clin Neurora diol. 2015;2:157-165.

6. Morrissey SP, Miller DH, Kendall BE, et al. The significance of brain magnetic resonance imaging abnormalities at presenta $\neg$ tion with clinically isolated syndromes suggestive of multiple sclerosis. A 5-year follow-up study. Brain. 1993;116(1):135-146.

7. Pitt D, Boster A, Pei W, et al. Imaging cortical lesions in multiple sclerosis with ultra-high-field magnetic resonance imaging. Arch Neurol. 2010;67(7):812-818

8. Traboulsee AL, Li DK. The role of MRI in the diagnosis of multiple sclerosis. Adv Neurol. 2006;98:25-146.

9. Harrison DM, Li X, Liu H, et al. Lesion heterogeneity on high-field sus $\neg$ ceptibility MRI is associated with multiple sclerosis severity. AJNR Am J Neuroradiol. 2016;37(8):1447-1453.

10. Alexander RW. Understanding adipose-derived stromal vascular fraction (AD-SVF) cell biology and use on the basis of cel $\neg$ lular, chemical, structural and paracrine components: a concise review. J Prolother. 2014;4:e855-e869.

11. Alexander RW. Biocellular regenerative medicine: use of adi $\neg$ posederived stem/stromal cells and it's native bioactive matrix. Phys Med Rehabil Clin N Am. 2016;27(4):871-891.

12. Luessi F, Siffrin V, Zipp F. Neurodegeneration in multiple sclerosis: novel treatment strategies. Expert Rev Neurother. 2012;12(9):1061-1076.

13. Ormerod IE, Miller DH, McDonald WI, et al. The role of NMR imaging in the assessment of multiple sclerosis and isolated neurological lesions. A quantitative study. Brain. 1987;110(6):1579-1616.

14. Tintoré $\mathrm{M}$, Rovira A, Martínez MJ, et al. Isolated demyelinat $\neg$ ing syndromes: comparison of different MR imaging criteria to predict conversion to clinically definite multiple sclerosis. AJNR Am J Neuroradiol. 2000;21(4):702-706.

15. Preziosa P, Rocca MA, Pagani E, et al. Structural MRI correlates of cognitive impairment in patients with multiple sclerosis: a multicenter study. Hum Brain Mapp. 2016;37(4):1627-1644.

16. Forbes FDS, Garcia LD, Barillot C, et al. A weighted mult-sequence markov model for brain lesion segmentation: France; 2010.

17. Garcia LD, Lecoeur J, Arnold DL, et al. Multiple sclerosis lesion segmentation using an automatic multimodal graph cuts. Med Image Comput Comput Assist Interv. 2009;12(2):584-591.

18. Traboulsee A, Simon JH, Stone L, et al. Revised recommenda $\neg$ tions of the consortium of MS centers task force for a stan-dardized MRI protocol and clinical guidelines for the diagnosis and follow-up of multiple sclerosis. AJNR Am J Neuroradiol. 2016;37(3):394-401. 